\title{
Efecto de la concentración de sulfato de sodio en la viscosidad y difusividad térmica de barbotinas de arcillas rojas
}

\author{
\begin{tabular}{l|l|l|l} 
V. Useche & G. Peña Rodríguez \\
& I. Segura & | L. Gualdrón
\end{tabular}
}

Recibido:

20 de marzo de 2009

Aceptado:

28 mayo de 2009
50

${ }^{1}$ Maestría en Física Universidad de Pamplona, Departamento de Física UFPS. Cúcuta Norte de Santander.

E-mail: vjuliouseche@hotmail.com ${ }^{2}$ Grupo de investigación en Tecnología Cerámica - GITEC Departamento De Física- UFPS. Cúcuta Norte de Santander E-mail: ggabrielp@yahoo.com. ${ }^{3}$ Asesor Técnico. Cerámica Italia S.A. ${ }^{4}$ Departamento de Física. Universidad de Pamplona. Norte de Santander.

\section{Resumen}

Se reporta el comportamiento de la viscosidad cinemática $(\sigma)$ y la difusividad térmica $(\alpha)$ de barbotinas de arcillas rojas utilizadas por la empresa Cerámica Italia S.A en la elaboración de polvos de arcillas atomizadas (spray dried), en función de la concentración de sulfato de sodio $\left(\mathrm{Na}_{2} \mathrm{SO}_{4}\right)$. Las barbotinas presentan un porcentaje en peso de sólido (arcilla) del $64 \%$ y un 36\% de agua (líquido). Para mantener condiciones de densidad similares (1.689 $\pm 0.007 \mathrm{~g} /$ $\mathrm{cm}^{3}$ ) y solo cambiar la viscosidad de la barbotina se realizó una curva de adición de $\mathrm{Na}_{2} \mathrm{SO}_{4}$ a la barbotina, desde 0,018\% hasta $0,153 \%$ en peso, para la medida de la viscosidad se usó el viscosímetro BROOKFIELD-DV-II+Pro; la densidad ( $\rho)$ se determinó mediante picnómetro metálico de $100 \mathrm{~cm}^{3}$; la difusividad térmica se hallo utilizando el sensor SH-2 del sistema KD2-Pro, el cual consiste en dos agujas de acero inoxidable separadas $6 \mathrm{~mm}$, una que funciona como fuente de calor y la otra como sensor de temperatura. La composición química usando fluorescencia de Rayos $X$ en porcentaje en peso de las arcillas reporta la presencia mayoritarias de $\mathrm{SiO}_{2}(64,4 \%), \mathrm{Al}_{2} \mathrm{O}_{3}(21,8 \%)$ y $\mathrm{Fe}_{2} \mathrm{O}_{3}$ (5,4\%). Los resultados reológicos indican que la viscosidad se incrementa cuando la concentración de sulfato de sodio aumenta en la muestra, presentándose un cambio en el estado de dispersión de las partículas, ya que las suspensiones manifiestan un comportamiento desde fluidas hasta alcanzar una mayor viscosidad. La difusividad térmica en función de la concentración de $\mathrm{Na}_{2} \mathrm{SO}_{4}$ mostró una desviación promedio del $3.5 \%$ reportándose un valor mínimo de $0.00330 \mathrm{~cm}^{2} / \mathrm{s}$ y uno máximo de 0.00375 $\mathrm{cm}^{2} / \mathrm{s}$. Los números de Prandtl $\left(P_{r}=\frac{\sigma}{\alpha}\right)$ encontrados fueron mucho mayor que uno $\left(P_{r}>>1\right)$ lo cual indica que la capa limite térmica es mucho menor que la capa límite hidrodinámica.

Palabras clave: Viscosidad, difusividad térmica, números de Prandtl, fluorescencia de rayos $X$, sulfato de sodio.

\section{Abstract}

We reports the thermal diffusivity $(\alpha)$ and kinematic viscosity $(\sigma)$ of red-clay suspensions used by Ceramica Italia SA. company in the productions of spraydried clay powders, in function of sodium sulphate concentration. The samples are formed in percentage in weight of a $64 \%$ of clay (solid) and a $36 \%$ of water 
(liquid). It is made an addition curve of $\mathrm{Na}_{2} \mathrm{SO}_{4}$ from $0,018 \%$ to $0,153 \%$ in weight, in order to maintain similar density conditions (1.689 \pm 0.007 $\left.\mathrm{g} / \mathrm{cm}^{3}\right)$ and only change the viscosity of the sample. The dynamic viscosity $(\mu)$ is measured using the viscosimeter BROOKFIELD-DV-II+Pro. Density $(\rho)$ is determined by a metallic pycnometer at $100 \mathrm{~cm}^{3}$. The thermal diffusivity is found by a SH-2 Sensor of the KD2-Pro System, which consists of 2 stainless steel needles $6 \mathrm{~mm}$ separated, one is functioning as a source of heat and the other one is as a sensor of temperature. X-Rays fluorescence is used to found the chemical composition it reports the presence of $\mathrm{SiO}_{2}(64,4 \%), \mathrm{Al}_{2} \mathrm{O}_{3}$ $(21,8 \%)$ y $\mathrm{Fe}_{2} \mathrm{O}_{3}(5,4 \%)$ mainly. The rheological results indicate that the viscosity increases when the concentration of sodium sulfate increases in the sample, appearing a change in the state of dispersion of particles; since the suspensions demonstrate a behavior from fluid to reach a higher viscosity. The thermal diffusivity depends on the concentration of $\mathrm{Na}_{2} \mathrm{SO}_{4}$ that showed an average deviation of $3.5 \%$ and reported a minimum value of 0.00330 $\mathrm{cm}^{2} / \mathrm{s}$ and a maximum value of $0.00375 \mathrm{~cm}^{2} / \mathrm{s}$. The Prandtl Numbers ( $P_{r}=\frac{\sigma}{\alpha}$ ) found for each concentration of $\mathrm{Na}_{2} \mathrm{SO}_{4}$ used, which were very much greater that one $(\operatorname{Pr}>>1)$, which indicates the thermal boundary layer is much smaller than the hydrodynamic boundary layer.

Keywords: Viscosity, Thermal diffusivity, Prandtl Numbers, X-Rays fluorescence, Sodium Sulfate.

\section{Introducción}

$\mathrm{L}$ as arcillas son materia prima que presentan múltiples aplicaciones tecnológicas. Se utiliza tanto para la elaboración de cerámica tradicional como para nuevos materiales (cerámicas avanzadas) útiles en la actualidad. En la industria de la construcción esta materia prima juega un papel importante, ya que con ésta se elaboran los distintos tipos de ladrillo, tejas, tubos, enchapes, pisos, los cuales deben cumplir con especificaciones técnicas de acuerdo a su uso [1]. Es un mineral complejo constituido por alumino-silicatos hidratados, comúnmente acompañados de otros minerales. Estructuralmente están constituidos por láminas de tetraedros $\mathrm{SiO}_{4}$ y octaedros $\mathrm{A}_{1} \mathrm{O}_{6}$. La combinación de estas láminas forman diferentes tipos de estructuras arcillosas que a su vez dan origen a paquetes, que al combinarse generan las placas de mineral arcilloso [2]. Físicamente se considera un coloide, de partículas extremadamente pequeñas y superficie lisa, donde sus propiedades derivan de su tamaño de partícula (inferior a $2 \mu \mathrm{m}$ ), su morfología laminar (filosilicatos) y las sustituciones isomórficas, que dan lugar a la aparición de carga en las láminas y a la presencia de cationes débilmente ligados en el espacio interlaminar [3]. Se caracteriza por adquirir plasticidad al ser mezclada con agua y se endurece al someterse a temperaturas por encima de $800^{\circ} \mathrm{C}$. Se utiliza en muchos procesos industriales, como en la industria del papel, producción de cemento e industrias químicas [4].

Las propiedades físicas más importantes de las arcillas son: (1) plasticidad, que permite que sea moldeada; (2) resistencia a compresión, tensión o cizallamiento; (3) retracción tanto en secado como en cocción; (4) temperatura de cocción y vitrificación; y (5) color de calcinación, que se debe principalmente a los óxidos de hierro presentes. La composición y 
su naturaleza determinan el uso y el valor de la arcilla[5].

Por otra parte, la viscosidad es una propiedad hidrodinámica de enorme importancia en los cálculos de ingeniería, la cual define el comportamiento reológico de un fluido, representando la resistencia que opone un fluido a la fluencia [6]. En el caso de las barbotinas o suspensiones de arcillas, los datos de la literatura disponible son muy escasos y no se han reportado datos de las mismas en la literatura técnica nacional. Las propiedades reológicas de un fluido dependen de factores como la composición química, humedad, distribución de tamaño de partícula, relación entre el contenido de fase sólida y liquida, temperatura, etc., [7]

El conocimiento de las propiedades reológicas y térmicas, es de vital importancia en la preparación de barbotinas o suspensiones ya que permiten optimizar las etapas de proceso en la fabricación de productos cerámicos. Las barbotinas y las suspensiones de arcilla en agua son fluidos que no se comportan como el agua u otros fluidos. La fluidez o viscosidad se pueden modificar con el agregado de electrolitos o defloculantes tales como silicato de sodio, hexametafosfato de sodio, carbonato de sodio, poliacrilatos, etc. [8].

Por lo general, las arcillas presentan también una característica particular llamada tixotropía que es el aumento de la viscosidad a través del tiempo. Una curva de defloculación se construye sobre dos ejes cartesianos en donde a uno se le asigna el valor de la viscosidad y en el otro la cantidad de defloculante agregado [9]. Las curvas dan una idea de cuan defloculada esta la barbotina y de la cantidad de defloculante a agregar si se quiere mantener siempre la misma viscosidad. A medida que se va agregando defloculante la fluidez va aumentando hasta un punto en el que comienza a disminuir y pasado ese límite se produce el fenómeno inverso que es la floculación. La adición o presencia de iones floculantes provoca el aumento de la viscosidad.
La razón entre viscosidad cinemática y la difusividad térmica define el número de Prandtl, el cual proporciona una medida de la efectividad relativa del transporte de momento y energía por difusión en las capas límite hidrodinámico y térmico, respectivamente [10].

En este trabajo, se presenta el estudio de la viscosidad y difusividad térmica en barbotinas de arcillas rojas usadas en la elaboración de polvos cerámicos por spray dried, utilizadas por la empresa Cerámica Italia S.A. en la fabricación de baldosas esmaltadas para piso y pared.

\section{Materiales y métodos}

Las muestras de barbotina fueron suministradas por la empresa Cerámicas Italia, S.A., ubicada en la ciudad de Cúcuta, Departamento Norte de Santander. Esta barbotina esta preparada con arcillas rojas naturales de la zona metropolitana de Cúcuta y se prepara por molienda vía húmeda en molino de bolas, usando como defloculante silicato de sodio y poliacrilato de sodio, la suspensión obtenida se tamiza por malla 80 y se usa posteriormente en la preparación de polvos para prensado de baldosas.

La relación sólido (arcilla) - líquido (agua), se determinó tomando una masa de 100 gramos de barbotina, se sometió a un proceso de secado a una temperatura de 110 ${ }^{\circ} \mathrm{C}$, por un tiempo de 24 horas usando estufa de circulación forzada marca Gabrielli ${ }^{\circledR}$. Para hallar la densidad $(\rho)$ de la barbotina se usó un picnómetro metálico con tapa de 100 $\mathrm{cm}^{3}$ y el $\mathrm{pH}$ se determinó según la Norma Técnica Colombiana NTC 5264 método A, que consiste en la medición del $\mathrm{pH}$ en una suspensión de arcilla-agua en relación 1:1 (peso/volumen).

El proceso de agitación de la muestra fue realizado con el agitador mecánico Velp Scientifica ${ }^{\circledR}$ a $1200 \mathrm{rpm}$ por tiempos de 5 minutos, mientras que para la determinación 
de la viscosidad dinámica $(\mu)$ se empleó el viscosímetro rotacional marca Brookfield ${ }^{\circledR}$, DV-II+Pro, utilizando la agujas 2,3 y 4 de acuerdo al corte con la muestra. La viscosidad cinemática $(\sigma)$ se calculó mediante la relación $\mu / \rho$. La difusividad térmica de la muestra se encontró usando el sensor $\mathrm{SH}-2$ del equipo $\mathrm{KD} 2{ }^{\circledR}$ Pro, el cual consta de dos agujas de 30 $\mathrm{mm}$ de largo y 1,4 $\mathrm{mm}$ de diámetro separadas 6 $\mathrm{mm}$, donde una de estas agujas funciona como fuente lineal de calor y la otra como el sensor de temperatura. La medida experimental de los parámetros anteriores se realizó en función de la concentración de sulfato de sodio $\left(\mathrm{Na}_{2} \mathrm{SO}_{4}\right)$, con el objeto de aumentar la viscosidad de la barbotina, agregándose cantidades constantes de 0,09 gramos iniciando con un valor mínimo de $0,18 \mathrm{~g}$ hasta un valor máximo de $1,53 \mathrm{~g}$, valor para el cual la viscosidad fue máxima. Para determinar el comportamiento de gelificación, inicialmente se tomó la muestra sin agregar sulfato de sodio y se dejó en el viscosímetro durante intervalos constantes de tiempo de 5 minutos, donde en cada uno de éstos se media la viscosidad hasta que ésta fuera constante. Posteriormente, a la muestra se le agregó cantidades constantes de 0.18 gramos de sulfato de sodio, empezando desde 0.27 hasta 1.17 gramos por cada kilogramo de barbotina y se determinó la viscosidad para cada caso en intervalos de 5 minutos. La composición química se determinó usando espectrómetro de fluorescencia de rayos $\mathrm{X}$ de energía dispersa Shimadzu EDX 800 HS, con detector de estado sólido $\mathrm{Si}(\mathrm{Li})$ y fuente de Rodio.

\section{Resultados y discusión.}

En la tabla 1, se presentan los valores obtenidos experimentalmente en función de la cantidad en gramos de sulfato de sodio agregada a la barbotina por cada kilogramo de ésta. Se observa, que la densidad prácticamente se mantuvo constante, obteniéndose un valor promedio de 1,689 \pm $0.007 \mathrm{~g} / \mathrm{cm}^{3}$, evidenciando que el $\mathrm{Na}_{2} \mathrm{SO}_{4}$ no influye de manera apreciable en la densidad de la barbotina; también se aprecia que al incrementar la concentración de $\mathrm{Na}_{2} \mathrm{SO}_{4}$ la viscosidad dinámica $(\mu)$ aumenta, es decir, cuando se toma una muestra de barbotina sin agregarle $\mathrm{Na}_{2} \mathrm{SO}_{4}$, ésta tiene un valor de 1166,7 cp y al agregarle 1,53 gramos de $\mathrm{Na}_{2} \mathrm{SO}_{4}$ la viscosidad dinámica presenta un valor de 6407 cp, elevándose en un $400 \%$, comportamiento esperado ya que esta sal tiene efecto floculante en la reología de las barbotinas, mostrando un cambio en el estado de dispersión de las

Tabla 1. Resultados experimentales de la densidad $(\rho)$, viscosidad dinámica $(\mu)$, viscosidad cinemática $(\sigma)$, difusividad térmica $(\alpha), \mathrm{pH}$, temperatura y número de Prandtl $(\operatorname{Pr})$

\begin{tabular}{|cccccccc|}
\hline $\begin{array}{c}\mathrm{Na} 2 \mathrm{SO} 4 \\
(\mathrm{~g})\end{array}$ & $\begin{array}{c}\rho \\
\left(\mathrm{g} / \mathrm{cm}^{3}\right)\end{array}$ & $\begin{array}{c}\mu \\
(\mathrm{cp})\end{array}$ & $\begin{array}{c}\sigma \\
\mathrm{cm}^{2} / \mathrm{s}\end{array}$ & $\begin{array}{c}\alpha \\
\left(\mathrm{cm}^{2} / \mathrm{s}\right)\end{array}$ & $\begin{array}{c}\mathrm{pH} \\
\text { Temperatura } \\
{ }^{\circ} \mathrm{C}\end{array}$ & $\begin{array}{c}\text { Número } \\
\text { Prandtl }\end{array}$ \\
\hline 0.000 & 1.686 & 1166.7 & 6.9 & 0.00342 & 8.6 & 24.1 & 2023.5 \\
0.180 & 1.680 & 1192.0 & 7.1 & 0.00358 & 8.9 & 25.4 & 1981.9 \\
0.270 & 1.681 & 1240.0 & 7.3 & 0.00330 & 9.0 & 25.8 & 2236.0 \\
0.360 & 1.680 & 1320.0 & 7.8 & 0.00352 & 8.7 & 26.3 & 2232.0 \\
0.450 & 1.681 & 1700.0 & 10.1 & 0.00358 & 8.7 & 26.3 & 2824.4 \\
0.540 & 1.683 & 1780.0 & 10.5 & 0.00352 & 8.8 & 26.4 & 3005.5 \\
0.630 & 1.685 & 1790.0 & 10.6 & 0.00351 & 8.9 & 26.5 & 3025.8 \\
0.720 & 1.685 & 1967.0 & 11.6 & 0.00358 & 8.9 & 26.6 & 3260.0 \\
0.810 & 1.687 & 2050.0 & 12.1 & 0.00363 & 8.6 & 26.7 & 3348.2 \\
0.900 & 1.687 & 2537.0 & 15.0 & 0.00352 & 8.8 & 26.6 & 4271.3 \\
0.990 & 1.690 & 3047.0 & 18.0 & 0.00372 & 8.8 & 26.8 & 4845.8 \\
1.080 & 1.695 & 3713.0 & 22.0 & 0.00375 & 8.7 & 26.9 & 5841.5 \\
1.170 & 1.696 & 4093.0 & 24.2 & 0.00347 & 8.7 & 26.8 & 6953.2 \\
1.260 & 1.698 & 4447.0 & 26.3 & 0.00373 & 8.7 & 26.7 & 7021.8 \\
1.350 & 1.699 & 5087.0 & 30.1 & 0.00359 & 8.8 & 26.7 & 8340.6 \\
1.440 & 1.699 & 6040.0 & 35.7 & 0.00374 & 8.7 & 26.7 & 9504.9 \\
1.530 & 1.702 & 6407.0 & 37.9 & 0.00371 & 8.5 & 26.6 & 10148.4 \\
\hline
\end{tabular}


partículas [11]. También se observa que el pH es básico, el cual prácticamente es constante, ya que su variabilidad respecto del promedio es aproximadamente del 1,4\%.

La viscosidad cinemática $(\sigma)$ se calculó utilizando la razón $\sigma=\mu / \rho$, para lo cual se tomaron los diferentes valores de $\mu$ y la densidad $(\rho)$ promedio hallada. En la figura 1, se presenta el mejor ajuste a los datos experimentales usando software OriginPro Ver. 7.0 de $\sigma(y)$ en función de la concentración de $\mathrm{Na}_{2} \mathrm{SO}_{4}(\mathrm{x})$, en ésta se observa que los datos hallados presentan un comportamiento que se ajustan a la función de la forma $y=y_{0}+A e^{-\left(x-x_{c}\right) / 2 w^{2}}$ donde $y_{0}$ corresponde a $\sigma$ para cero concentración de $\mathrm{Na}_{2} \mathrm{SO}_{4} \operatorname{con} \mathrm{A}, x_{c}$ y $w$ parámetros de ajuste. La función exponencial, indica que incrementos iguales del contenido de $\mathrm{Na}_{2} \mathrm{SO}_{4}$ producen incrementos variables y crecientes del valor de $\sigma$ a medida que aumenta la concentración de $\mathrm{Na}_{2} \mathrm{SO}_{4}$ en la barbotina. Esto indica el efecto floculante y la influencia de la concentración de esta sal $\left(\mathrm{Na}_{2} \mathrm{SO}_{4}\right)$.

Figura 1. Ajuste multiparamétrico de la viscosidad cinemática $(\sigma)$ en función de la concentración de $\mathrm{Na}_{2} \mathrm{SO}_{4}$ agregado a la barbotina, la línea sólida representa el mejor ajuste a los datos experimentales usando la expresión $y=y_{0}+A e^{-\left(x-x_{c}\right) / 2 w^{2}}$

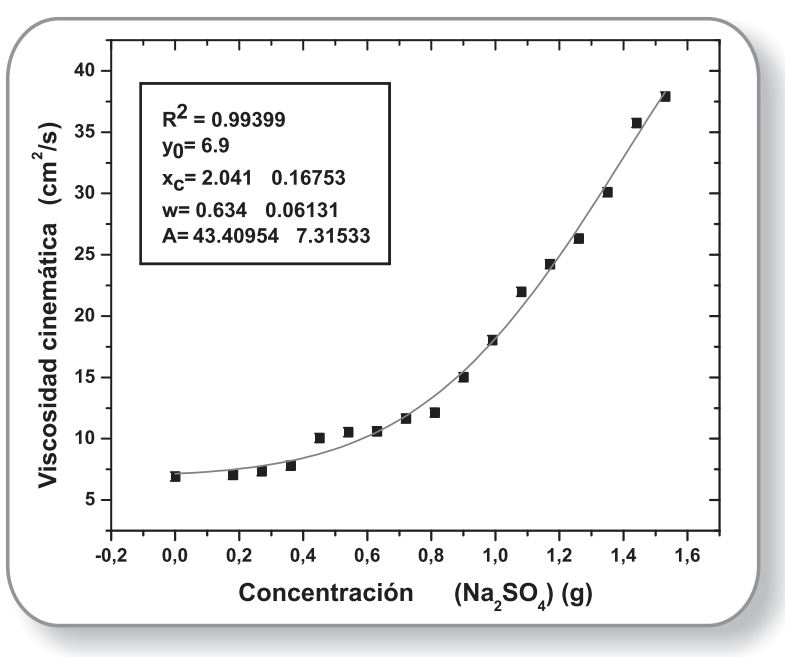

Al cambiar la concentración de $\mathrm{Na}_{2} \mathrm{SO}_{4}$ en la barbotina, se puede observar en los datos experimentales que la difusividad térmica (a) no sufre una variación considerable, obteniéndose un valor promedio $0.00358 \pm$ $0.00012 \mathrm{~cm}^{2} / \mathrm{s}$ para una temperatura promedio de $26.3 \pm 0.7^{\circ} \mathrm{C}$, lo anterior permite inferir que la capa límite térmica $\left(\delta_{\mathrm{t}}\right)$ es mucho menor que la capa límite hidrodinámica $(\delta)$, lo cual se corrobora al analizar los números de Prandtl $\left(P_{r}\right)$, ya que éstos valores son muchos mayores que la unidad $\left(P_{r}>>1\right)$. De la definición de $P_{r}=\sigma / \alpha[12]$, se espera que el comportamiento de la difusividad térmica ( $\alpha$ ) en función de $\sigma / P_{r}$ se ajuste mediante la función identidad, lo cual se confirma tal como se aprecia en la figura 2.

Figura 2. Difusividad térmica $(\alpha)$ en función de la viscosidad cinemática normalizada con el número de Prandtl (Pr). La línea sólida representa el mejor ajuste a los datos experimentales con la función identidad.

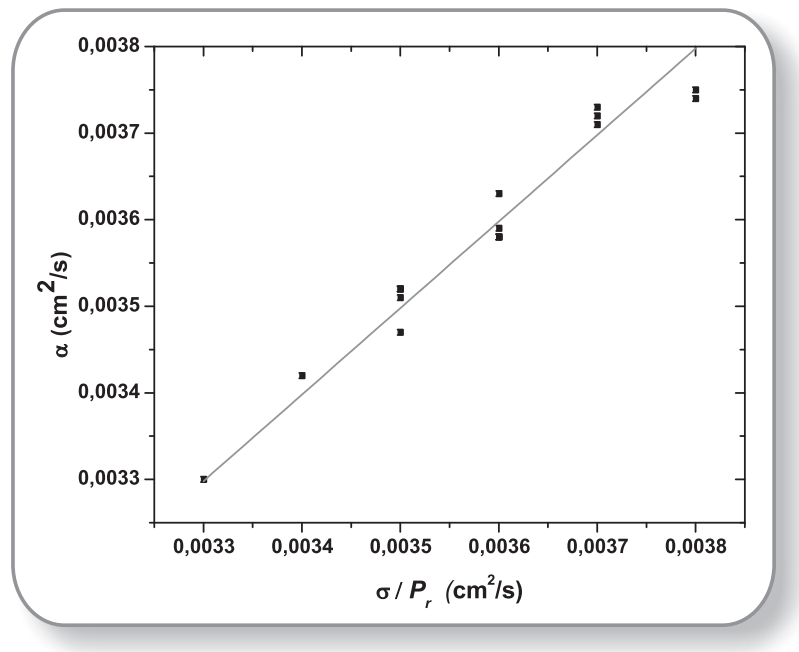

En la tabla 2, se muestran los valores obtenidos experimentalmente de la viscosidad dinámica $(\mu)$, en función de la concentración de sulfato de sodio agregada a la muestra y del tiempo de gelificación. El utilizar el $\mathrm{Na}_{2} \mathrm{SO}_{4}$ como floculante en la barbotina, hace que la viscosidad aumente debido al mecanismo de la floculación, donde el efecto del sulfato de sodio es disminuir la fuerza de repulsión entre las partículas de arcilla (Potencial Z) y aumentar la viscosidad de la barbotina. La figura 3, presenta la curva de gelificación de la barbotina, presentándose los valores de viscosidad dinámica expresados en centipoise (cp) en función del tiempo de gelificación en minutos. 
Efecto de la concentración de sulfato de sodio en la viscosidad y difusividad térmica de barbotinas de arcillas rojas

Tabla 2. Resultados experimentales de la viscosidad dinámica $(\mu)$ en función de la concentración de sulfato de sodio y el tiempo de gelificación.

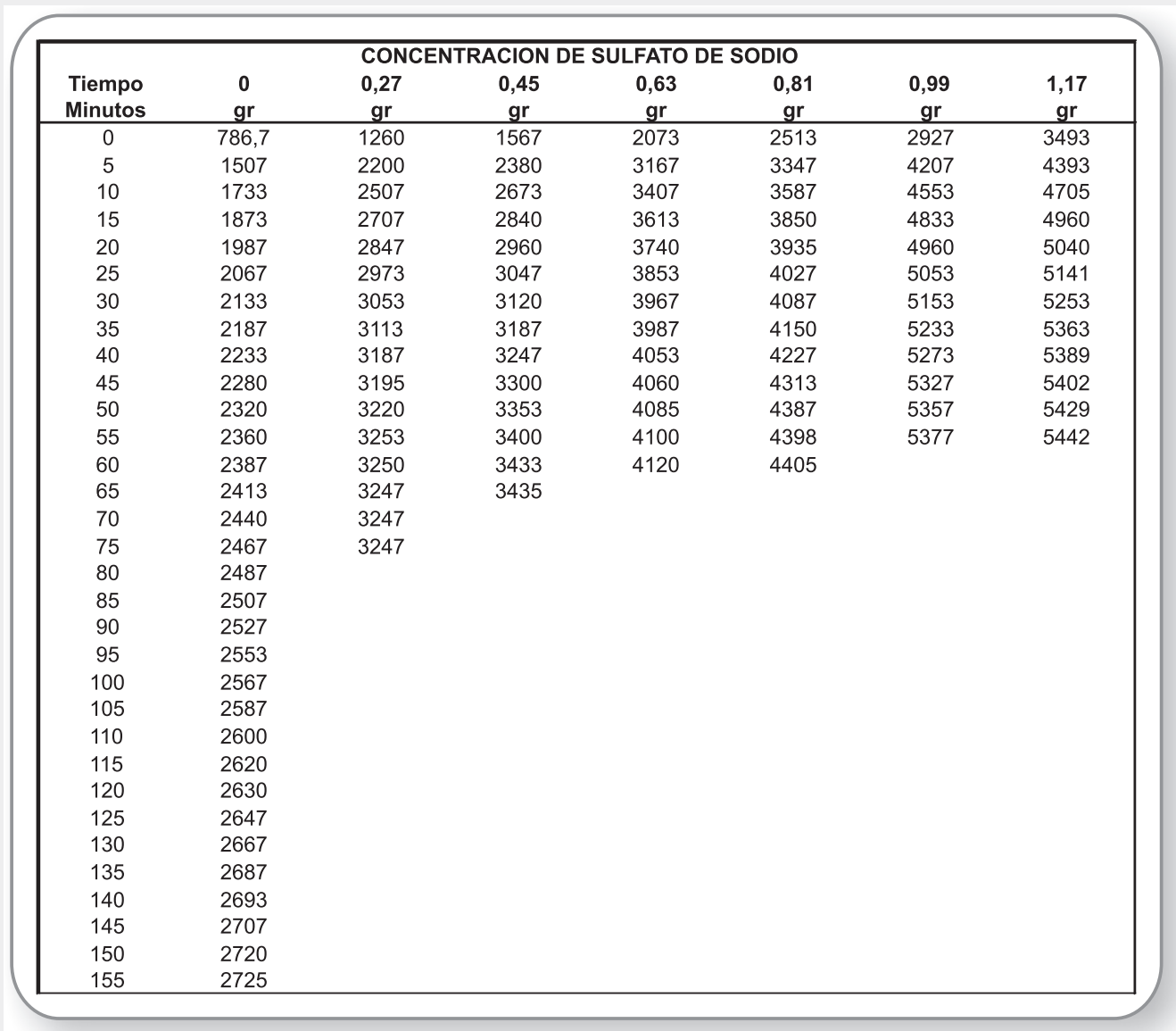

Figura 3. Curva de gelificación de la barbotina en función de la concentración de sulfato de sodio y el tiempo

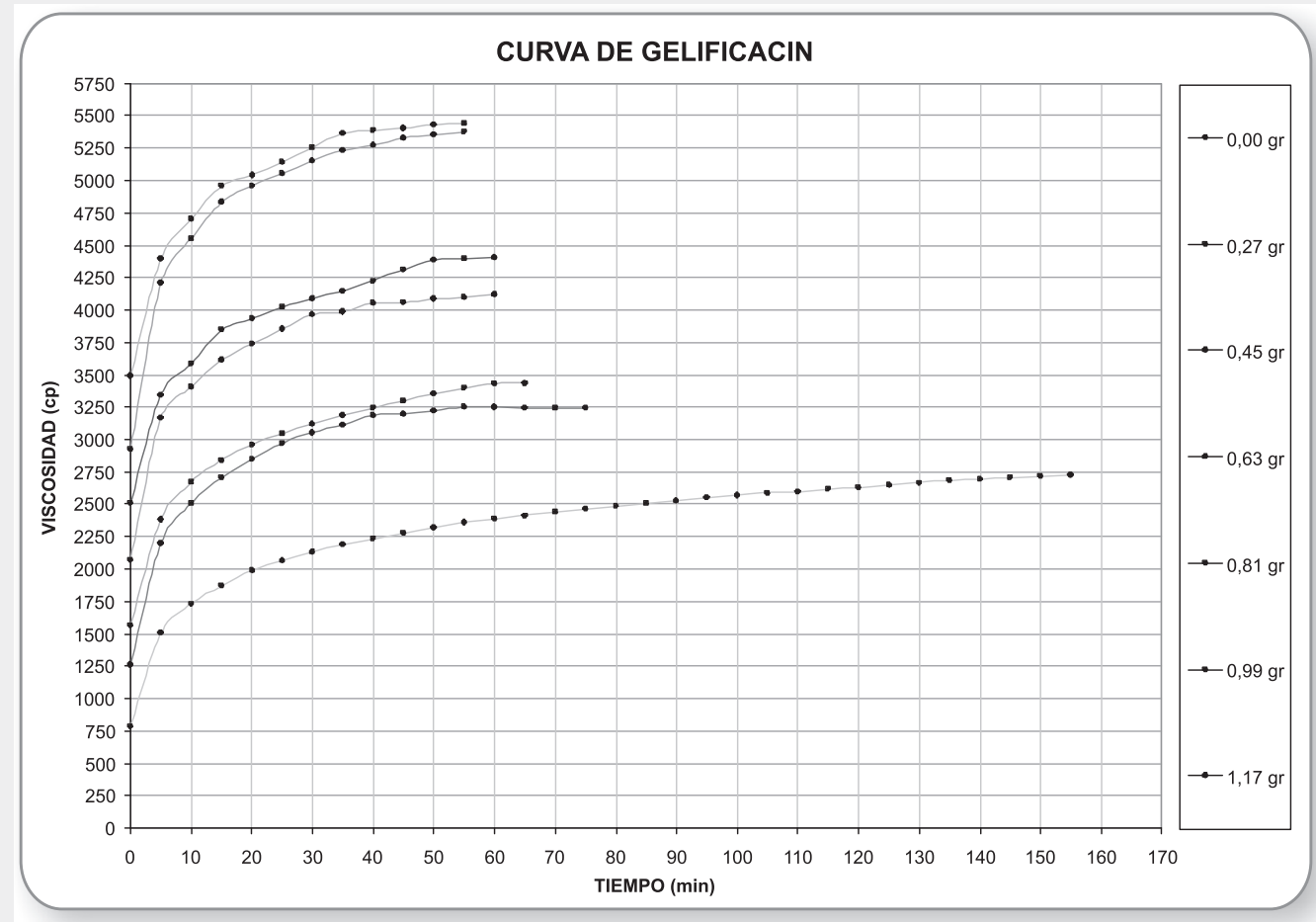


Al remitirnos a la figura anterior, se puede afirmar que la consistencia de la muestra aumenta y su fluidez disminuye, entendiéndose por consistencia, el grado de resistencia que ofrece la barbotina a fluir o a deslizarse. Las partículas coloidales en estado de reposo forman geles (de apariencia sólida), y cuando estos geles se someten a enérgicas agitaciones, se destruyen y forman fluidos viscosos. Si la agitación se detiene y la suspensión permanece inmóvil, se regeneran las características del gel original. Las partículas coloidales son atrapadas en una posición de equilibrio eléctrico cuando se forma el gel, balanceándose de este modo todas sus cargas, mediante la acción de los iones del medio, que son capaces de mantener a las partículas negativas de arcilla con cierta separación.

Tabla 3. Composición química en porcentaje en peso (\%Wt) de las arcillas usando FRX.

\begin{tabular}{|c|c|c|c|}
\hline ELEMENTO & \% Wt & ELEMENTO & $\%$ Wt \\
\hline $\mathrm{SiO}_{2}$ & 64.440 & $\mathrm{Na}_{2} \mathrm{O}$ & 0.145 \\
\hline $\mathrm{Al}_{2} \mathrm{O}_{3}$ & 21.820 & $\mathrm{~V}_{2} \mathrm{O}_{5}$ & 0.057 \\
\hline $\mathrm{Fe}_{2} \mathrm{O}_{3}$ & 5.354 & $\mathrm{MnO}$ & 0.053 \\
\hline $\mathrm{K}_{2} \mathrm{O}$ & 1.583 & $\mathrm{ZnO}$ & 0.022 \\
\hline $\mathrm{SO}_{3}$ & 1.355 & $\mathrm{ZrO}_{2}$ & 0.019 \\
\hline $\mathrm{TiO}_{2}$ & 0.908 & $\mathrm{SrO}$ & 0.014 \\
\hline $\mathrm{CaO}$ & 0.512 & $\mathrm{Rb}_{2} \mathrm{O}$ & 0.008 \\
\hline $\mathrm{MgO}$ & 0.477 & $\mathrm{Y}_{2} \mathrm{O}_{3}$ & 0.003 \\
\hline & & $\mathrm{CO}_{2}$ & 6.230 \\
\hline
\end{tabular}

La composición química en porcentaje en peso $(\% \mathrm{Wt})$ de las arcillas usando fluorescencia de rayos $\mathrm{X}$ de energía dispersa (FRX), se presenta en la tabla 3. En ésta, se aprecia que más del $86 \%$ de la muestra está constituida por sílice $\left(\mathrm{SiO}_{2}\right)$ y alúmina $\left(\mathrm{Al}_{2} \mathrm{O}_{3}\right)$, también se observa en menor concentración la presencia de óxidos de hierro, potasio, azufre, titanio, calcio y magnesio entre otros

\section{Conclusiones}

Paraunabarbotinaindustrialdearcillasrojas, con composición química mayoritaria de sílice y alúmina, y con una relación en peso sólido (arcilla) - líquido (agua) constante de 64,23 \pm $0,53 \%$ y $35,77 \pm 0,53 \%$ respectivamente, la viscosidad cinemática de las barbotinas, con la adición de sulfato de sodio, entre 0,018\% y $0.153 \%$, varió en forma exponencial según la expresión $y=y_{0}+A e^{-\left(x-x_{c}\right) / 2 w^{2}}$, mientras que la difusividad térmica prácticamente fue constante reportándose un valor promedio de $0.00358 \pm 0.00012 \mathrm{~cm}^{2} / \mathrm{s}$ para una temperatura promedio de $26.3 \pm 0.7{ }^{\circ} \mathrm{C}$, lo anterior era de esperarse pues no se encontró una variabilidad significativa en la densidad de las muestras, hallándose un valor promedio de 1,689 $\pm 0.007 \mathrm{~g} / \mathrm{cm}^{3}$. La relación entre la viscosidad cinemática y la difusividad térmica quedó definida por los números de Prandtl $\left(P_{r}\right)$, encontrándose que $P_{r}>>1$, indicando que la capa limite térmica es mucho menor que la capa límite hidrodinámica $(\delta \mathrm{t}<<$ $\delta)$, luego la rapidez con la que se difunde el calor es mucho menor que la cantidad de movimiento. Se puede inferir que al aumentar la concentración de sulfato de sodio aumenta la viscosidad, y ésta depende del tiempo de reposo y del nivel de agitación como se pudo observar en las curvas de floculación y curvas de gelificación. Los resultados anteriores, serán utilizados en los procesos de elaboración de los polvos de arcillas atomizados (spray dried), los cuales son utilizados en la fabricación de cerámica esmaltada para piso y pared por procesos de prensado.

\section{Agradecimientos}

Al Centro de Investigación en Materiales Cerámicos de la UFPS por su colaboración en el préstamo de los equipos y laboratorios, a la Tecnóloga Química Tatiana Mendoza Calderón por su asistencia técnica en la preparación de las muestras.

\section{Bibliografía}

[1] E. García, M. Suárez. Curso Ingeniería de Minas y Petróleos. Las arcillas: propiedades y usos. http://www.uclm. es/users/higueras/yymm/Arcillas.htm. Consultado el 18 de marzo de 2009 
[2] J. Bernal, H. Cabezas, C. Espitia, J. Mojica, J. Quintero. Análisis próximo de arcillas para cerámica. Rev. Acad. Colomb. Cienc. Vol. 27, 2003, pp. 569-578

[3] Barnes, H.A Hutton JF \& Walters K. An Introduction to Rheology, Elsevier Science Publish, Amsterdam, 1989.

[4] Barba A., Beltrán V., Feliu C., Garcia J., Ginés F., Sánchez E. y Sanz Vicente. Materias primas para la fabricación de soportes de baldosas cerámicas. Segunda edición. Instituto de Tecnología Cerámica ( ITC). Castellón (España). 2002, p. 37.

[5] M.A. Hoyos, J. Casas, J. Guijarro, J. L. Martín de Vidales. Boletín geológico y minero. ISSN 0366-0176, Vol. 101, № 2, 1990, pp. 121-130.

[6] Pinzón B, Jorge Alejo. SUSPENSIONS VISCOSITY OF A NATURAL AND ORGANOPHILIC BENTONITE. Rev. Colomb.Quim., Jul. /Dec. 2006, Vol. 35, № 2, pp. 225-232.

[7] J. Pinzón, J. Requena. Caracterización y estudio reológico de una bentonita colombiana. Bol. Soc. Esp. Ceram. Vidrio. 1996, 35(5), 337-344.

[8] R. Asunción. Foro de Cerámica Foro de Vidrio. http://www.manises. com-forum-topic.aspTOPIC I D $=1351 \&$ A R C H I VE $=$. url. Consultado el 16 de febrero de 2009

[9] Jazayer, S.H.; Salem. A.; Timellini, G.; Rastell, E.; Albertazzi, A. Influencia de nefelina sienita y del tiempo de molienda en las propiedades reológicas de una barbotina de porcelana gres. Boletín de la Sociedad Española de Cerámica y Vidrio, 2003, JUL-AGO, 42 (4), pp. 209-213
[10] Ramos. Capa Límite Superficial. Física Ambiental. Tema 6. http://www2.uah. es/gifa/documentos/FA/Transparencias fa/tema6 fa.pdf. Consultado el 16 de febrero de 2009.

[11] Pinzón-Bello J.; Requena-Balmaseda J. Caracterización y estudio reológico de una bentonita colombiana. Bol. Soc. Esp. Ceram. Vidrio. 1996; 35(5):337344.

[12] Ramos, Capa Límite Superficial. Física Ambiental. Tema 6. www.uah.es/gifa/ documentos/FA/Transparencias_fa/ tema6 fa.pdf. Consultado el $16^{-}$de febrero de 2009. 